\title{
A catalogue of compact radio sources in and behind the Large Magellanic Cloud ${ }^{\star}$
}

\author{
M. Marx ${ }^{1}$, J.M. Dickey ${ }^{1,2}$, and U. Mebold ${ }^{1}$ \\ 1 Radioastronomisches Institut der Universität Bonn, Auf dem Hügel 71, D-53121 Bonn, Germany \\ 2 Department of Astronomy, University of Minnesota, Minneapolis, Minnesota 55455, U.S.A.
}

Received January 19; accepted March 10, 1997

\begin{abstract}
We present the results of a continuum snapshot survey of a $3^{\circ} \times 4^{\circ}$ region of the Large Magellanic Cloud including the area of the giant molecular cloud and the 30 Doradus nebula. The observations have been carried out with the Australia Telescope Compact Array $(\mathrm{ATCA})^{1}$ at 1.4 and $2.4 \mathrm{GHz}$. Most fields are complete to about $6 \mathrm{mJy}$ peak flux density at $1.4 \mathrm{GHz}$ and to about $3 \mathrm{mJy}$ at $2.4 \mathrm{GHz}$. The positions, peak and integral flux densities of 113 compact $\left(<54^{\prime \prime}\right)$ sources detected at $1.4 \mathrm{GHz}$ and of 70 sources $\left(<34^{\prime \prime}\right)$ detected at $2.4 \mathrm{GHz}$ are presented. Positions are accurate to about $3^{\prime \prime}$ and peak flux densities are accurate to about $10 \%$ or better, depending on the source position relative to the pointing centers. 32 of the sources detected at $1.4 \mathrm{GHz}$ are coincident with $\mathrm{H} \alpha$ objects in the catalogue of Davies et al.; these are possibly intrinsic to the LMC. However, we suppose that most are background objects, since the number vs. flux agrees with predictions of extragalactic source counts from other surveys.
\end{abstract}

Key words: Magellanic Clouds — radio continuum: galaxies — surveys - HII regions methods: statistical

\section{Introduction}

The radio-continuum emission of the LMC has been investigated numerous times at several frequencies. The first catalogue of radio sources in the LMC (MC catalogue) was obtained by McGee et al. (1972a,b). They observed the LMC with the Parkes radio telescope at $5 \mathrm{GHz}$ and

\footnotetext{
Send offprint requests to: M. Marx

* Tables 3 and 4 are also available electronically at the CDS via ftp cdsarc.u-strasbg.fr (130.79.128.5) or via http://cdsweb.u-strasbg.fr/Abstract.html

1 The Australia Telescope is funded by the Commonwealth of Australia for operation as a National Facility managed by CSIRO.
}

$2.7 \mathrm{GHz}$. As a part of the whole sky survey at $0.408 \mathrm{GHz}$ the LMC has been observed by Clark et al. (1976) using the Molonglo radio telescope. They detected 227 sources presented in the MC4 catalogue. Recent surveys of the LMC include the observations with Parkes at several frequencies $(1.4 \mathrm{GHz}, 2.45 \mathrm{GHz}, 4.75 \mathrm{GHz}$ and $8.55 \mathrm{GHz}$ ) (Filipovic et al. 1995) revealing 469 discrete sources, the PMN southern sky survey at $4.85 \mathrm{GHz}$ (Wright et al. 1994) and the MOST survey at $0.843 \mathrm{GHz}$ (Mills et al. 1984a,b; Ye et al., in preparation).

The Australia Telescope Compact Array (ATCA) offers the possibility of surveying compact sources in and behind the LMC with much higher spatial resolution than has been possible previously. Here we present the results of a short (12 min per field) ATCA continuum survey ("snapshot" survey) at $1.4 \mathrm{GHz}$ and $2.4 \mathrm{GHz}$ in the region of the giant molecular cloud and 30 Doradus.

This catalogue of compact (subarcminute) sources is useful for several purposes. Compact sources are needed to search for neutral-hydrogen $21 \mathrm{~cm}$-line absorption. To study the cool $(T \lesssim 100 \mathrm{~K})$ atomic gas in the LMC an interferometer is necessary, because it acts as a spatial filter, removing the extended emission which confuses the absorption measurement. Baselines longer than about $800 \mathrm{~m}$ (3000 wavelengths) are needed (Dickey et al. 1994; Marx et al. in preparation), so the background sources must be smaller than about $50^{\prime \prime}$. The deepest survey of the LMC with sufficiently high angular resolution, the MOST survey $\left(0.75^{\prime}\right)$, is not sensitive enough to include all sources which are useful for an HI-absorption study of the LMC. A list of point sources complete to about $6 \mathrm{mJy}$ peak flux density at $1.4 \mathrm{GHz}$ and to about $3 \mathrm{mJy}$ at $2.4 \mathrm{GHz}$ is given by the present ATCA snapshot survey.

The compact radio sources in directions towards the LMC are also interesting in themselves. Most compact radio sources are background objects like quasars or Seyfert galaxies. But there are also compact objects located within the LMC. These intrinsic sources are of peculiar interest, as they might be compact HII regions (Habing \& Israel 1979), young supernova remnants or planetary nebulae. 
Table 1. Field centre positions and completeness levels

\begin{tabular}{|c|c|c|c|c|c|c|c|c|c|}
\hline \multirow{3}{*}{$\begin{array}{l}\text { Field No. } \\
\text { 1D1 }\end{array}$} & \multirow{2}{*}{\multicolumn{2}{|c|}{ 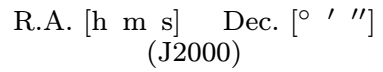 }} & \multicolumn{2}{|c|}{$C$-level [mJy] } & \multirow{3}{*}{$\begin{array}{l}\text { Field No. } \\
\text { 1D5 }\end{array}$} & \multirow{2}{*}{\multicolumn{2}{|c|}{ 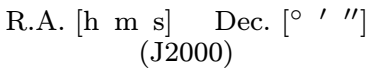 }} & \multicolumn{2}{|c|}{$C$-level [mJy] } \\
\hline & & & $21 \mathrm{~cm}$ & $13 \mathrm{~cm}$ & & & & \multicolumn{2}{|c|}{$21 \mathrm{~cm} \quad 13 \mathrm{~cm}$} \\
\hline & 052000.0 & -671500 & 7.5 & 5.5 & & 052000.0 & -691500 & 5.1 & 2.2 \\
\hline 2D1 & $05 \quad 2535.2$ & -671500 & 5.5 & 4.5 & 2D5 & 052535.2 & -691500 & 11.0 & 3.3 \\
\hline 3D1 & 053110.5 & -671500 & 5.5 & 2.0 & 3D5 & 053110.5 & -691500 & 4.0 & 2.0 \\
\hline 4D1 & 053646.1 & -671500 & 6.0 & 6.0 & 4D5 & 053646.1 & -691500 & 35.0 & 13.0 \\
\hline $5 \mathrm{D} 1$ & 054221.4 & -671500 & 3.0 & 2.0 & 5D5 & 054221.4 & -691500 & 13.0 & 6.6 \\
\hline 6D1 & 054752.0 & -671500 & 2.0 & 2.0 & 6D5 & 054752.0 & -691500 & 6.0 & 2.1 \\
\hline 1D2 & 052000.0 & -674500 & 8.0 & 3.0 & 1D6 & 052000.0 & -694500 & 6.0 & 5.2 \\
\hline 2D2 & $05 \quad 2535.2$ & -674500 & 5.5 & 3.1 & 2D6 & 052535.2 & -694500 & \multicolumn{2}{|c|}{ not complete } \\
\hline 3D2 & 053110.5 & -674500 & 9.0 & 8.7 & 3D6 & 053110.5 & -694500 & 6.0 & 2.5 \\
\hline $4 \mathrm{D} 2$ & 053646.1 & -674500 & 5.0 & 2.1 & $4 \mathrm{D} 6$ & 053646.1 & -694500 & 7.0 & 6.6 \\
\hline $5 \mathrm{D} 2$ & 054221.4 & -674500 & 5.0 & 1.9 & $5 \mathrm{D} 6$ & 054221.4 & -694500 & 12.0 & 4.1 \\
\hline $6 \mathrm{D} 2$ & 054752.0 & -674500 & 7.0 & 2.2 & 6D6 & 054752.0 & -694500 & 4.5 & 2.4 \\
\hline 1D3 & 052000.0 & -681500 & 5.5 & 2.0 & 1D7 & 052000.0 & -701500 & 3.0 & 4.6 \\
\hline 2D3 & 052535.2 & $-68 \quad 1500$ & 6.0 & 2.4 & $2 \mathrm{D} 7$ & 052535.2 & -701500 & 4.0 & 3.0 \\
\hline 3D3 & 053110.5 & -681500 & 3.8 & 2.3 & $3 \mathrm{D} 7$ & 053110.5 & -701500 & 4.5 & 2.4 \\
\hline 4D3 & 053646.1 & -681500 & 3.0 & 2.3 & $4 \mathrm{D} 7$ & 053646.1 & -701500 & 4.5 & 3.1 \\
\hline $5 \mathrm{D} 3$ & 054221.4 & -681500 & 8.0 & 4.0 & $5 \mathrm{D} 7$ & 054221.4 & -701500 & 3.0 & 3.0 \\
\hline 6D3 & 054752.0 & -681500 & 4.0 & 3.5 & $6 \mathrm{D} 7$ & $\begin{array}{lll}05 & 47 & 52.0\end{array}$ & -701500 & 5.0 & 3.4 \\
\hline 1D4 & 052000.0 & -684500 & 3.0 & 2.3 & 1D8 & 052000.0 & -704500 & 7.0 & 4.8 \\
\hline 2D4 & 052535.2 & -684500 & 3.5 & 2.5 & 2D8 & 052535.2 & -704500 & 14.0 & 7.4 \\
\hline $3 \mathrm{D} 4$ & 053110.5 & -684500 & 4.0 & 2.3 & $3 \mathrm{D} 8$ & 053110.5 & -704500 & 4.0 & 2.7 \\
\hline $4 \mathrm{D} 4$ & 053646.1 & -684500 & 8.0 & 2.6 & $4 \mathrm{D} 8$ & 053646.1 & -704500 & 4.5 & 2.9 \\
\hline $5 \mathrm{D} 4$ & 054221.4 & -684500 & 10.0 & 2.3 & $5 \mathrm{D} 8$ & 054221.4 & -704500 & 6.0 & 3.9 \\
\hline 6D4 & 054752.0 & -684500 & 11.0 & 5.2 & 6D8 & 054752.0 & -704500 & 7.0 & 3.4 \\
\hline
\end{tabular}

A list of compact radio sources is the first step toward identifying such objects.

\section{Observations and reduction}

\subsection{Observations}

The observations were carried out with the ATCA in July 1992 using the 6D configuration including baselines between $77 \mathrm{~m}$ and $5878 \mathrm{~m}$. The observed region extends from $5^{\mathrm{h}} 18^{\mathrm{min}}$ to $5^{\mathrm{h}} 50^{\mathrm{min}}$ in R.A. and $-71^{\circ}$ to $-67^{\circ}$ in Dec. (positions are in J2000). This area of 12 square degrees has been divided into $6 \times 8$ fields. The centre position of each of the 48 fields is given in Table 1 . Three observations were taken per field with a time seperation of about 4 hours. The total integration time was 12 minutes for each field. Observations at two frequencies, $1.38 \mathrm{GHz}$ and $2.378 \mathrm{GHz}$, have been carried out simultaneously. The angular resolution at $1.4 \mathrm{GHz}$ is $7^{\prime \prime}$ and at $2.4 \mathrm{GHz}$ it is $4^{\prime \prime}$. The primary beam has a HPW of $32^{\prime}$ at $1.4 \mathrm{GHz}$ and $22^{\prime}$ at $2.4 \mathrm{GHz}$. The bandwidth of $128 \mathrm{MHz}$ at each frequency has been separated into 32 spectral channels. The primary flux and bandpass calibrator was $1934-638(16.2 \mathrm{Jy}$ at $1.4 \mathrm{GHz}$ and $13 \mathrm{Jy}$ at $2.4 \mathrm{GHz}$ ), the secondary (phase and gain) calibrators were 0407-658 and 0252-712. For data reduction the Astronomical Image Processing System (AIPS) in the special ATCA version was used.

\subsection{The maps}

The images were made using the AIPS routine MX, which combines the Fourier Transform imaging with the deconvolution of the synthesized beam. The deconvolution process uses the clean algorithm of Clark (1980) with 500 iterations. We chose the inner 18 channels of the frequency band to compute the images using the technique of multifrequency synthesis.

The sparse $u v$-coverage of snapshot observations makes imaging difficult due to poor dynamic range in the maps. Extended emission is poorly sampled and cannot be adequately cleaned. We have tested several ways of selecting the data for mapping. Best results were obtained by restricting the data to baselines longer than $3 k \lambda$ to exclude the poorly sampled extended emission. In the region around 30 Doradus only baselines above $6 k \lambda$ are useful. We taper the uv data with a Gaussian weighting function with half width to $30 \%$ level of $15 k \lambda$ at $1.4 \mathrm{GHz}$ and $30 k \lambda$ at $2.4 \mathrm{GHz}$. This gives synthesized beamwidths of about $10^{\prime \prime}$ at $1.4 \mathrm{GHz}$ and $6^{\prime \prime}$ at $2.4 \mathrm{GHz}$ (see Table 2 ). The shape of the synthesized beam depends on the declination. The beam has a circular shape only for fields with declination between $-69.25^{\circ}$ and $-68.25^{\circ}$. For higher and lower declination, the shape of the synthesized beam is more and more elongated, in the extreme case (fields at $-67.25^{\circ}$ ) it is about $24^{\prime \prime} \times 8^{\prime \prime}$ at $1.4 \mathrm{GHz}$. The maps are $49^{\prime} \times 49^{\prime}$ at $1.4 \mathrm{GHz}$ and overlap slightly. At $2.4 \mathrm{GHz}$ the map size is $31^{\prime} \times 31^{\prime}$. The maps have an rms noise level between $0.4 \mathrm{mJy}$ and $1 \mathrm{mJy}$ at $1.4 \mathrm{GHz}$ and between $0.2 \mathrm{mJy}$ and 
$0.6 \mathrm{mJy}$ at $2.4 \mathrm{GHz}$. The theoretical $\mathrm{rms}$ values are 0.17 mJy at $1.4 \mathrm{GHz}$ and $0.23 \mathrm{mJy}$ at $2.4 \mathrm{GHz}$ based on receiver noise alone. The excess is due to sidelobes from confusing sources aggravated by our limited dynamic range.

Table 2. Imaging parameters

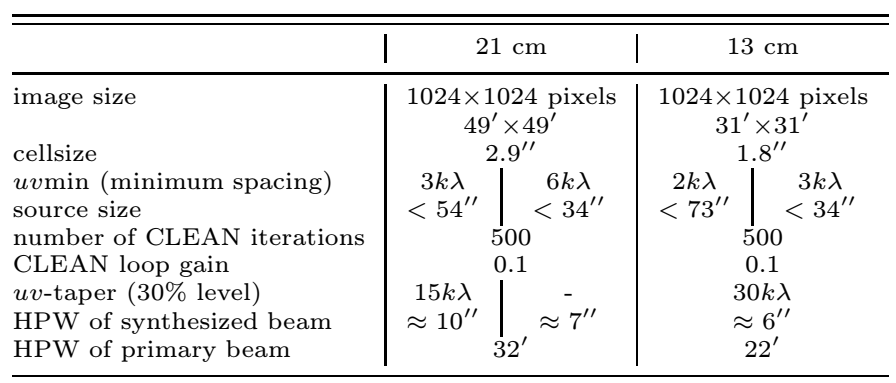

To correct the images for primary beam attenuation the program PBCOR was used with the standard AT primary beam shape. We tested this by comparing the peak flux densities of sources detected separately in different overlapping fields. We find that the ratio of the flux densities of the same source in two fields is independent of the difference of its distances from the pointing centres. Only for some very weak sources with a large distance $d$ (above $20^{\prime}$ ) from the field centre does this ratio differ much from one, indicating a higher uncertainty of the primary beam correction for $(d \cdot \nu)^{2}>780$.

A mosaic of the 48 final images is shown in the first figure. $3 \times 4$ fields of $49^{\prime} \times 49^{\prime}$ each have been combined with the AIPS routine LTESS which takes the primary beam attenuation into account. The resultant four mosaicing fields have then been combined with the task COMB, averaging the overlapping areas. The image shown is smoothed to about $1^{\prime}$ resolution.

\subsection{Source finding}

Very brief observations in the snapshot mode are ideal to study sources which are bright and compact, but the high sidelobe levels of beams synthesized from snapshots exacerbate the problems created by confusing sources. Some sidelobes cannot be completely eliminated in our cleaning process. The fields near 30 Dor are particularly compromised. Due to such sidelobe structure, the minimum detectable source flux is raised. We determined three criteria to decide which objects are real:

- The source must have signal exceeding 5 times the rms noise of the field, and

- the source must be stronger than any remaining sidelobe structure in the field, and

- the source has to be stronger than the absolute flux density value of the largest negative peak in the field.

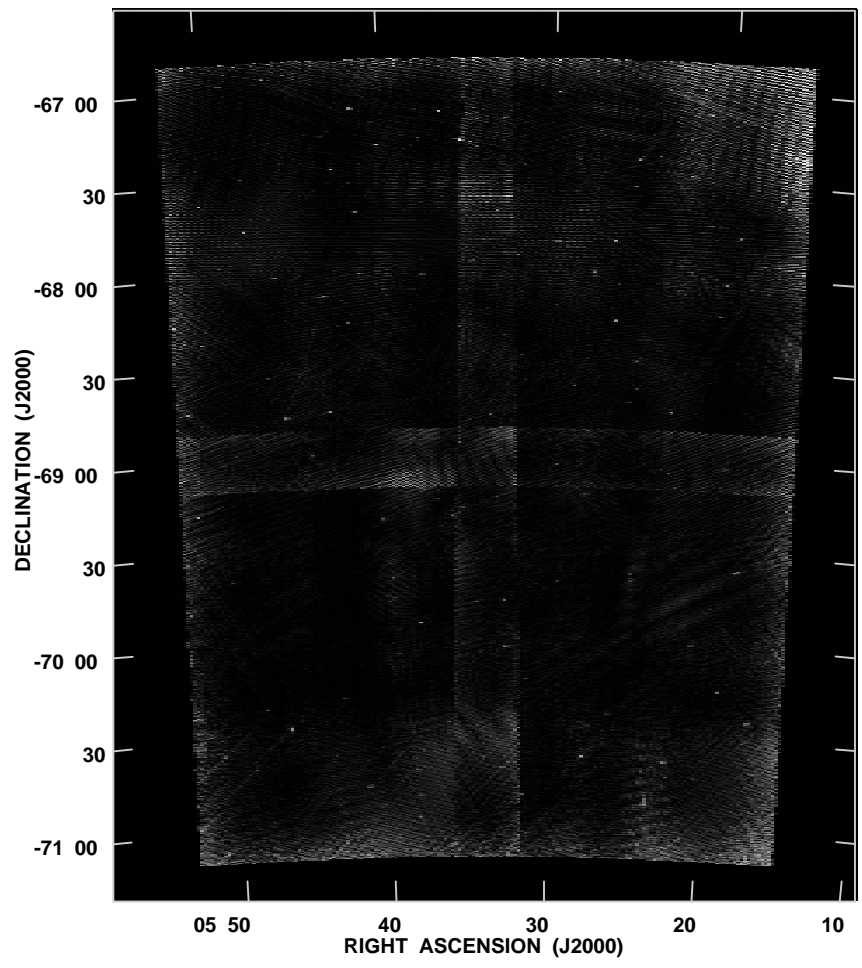

Fig. 1. Mosaic of all 48 images at $1.4 \mathrm{GHz}$. The cross feature is the overlapping area of the four combined LTESS-fields. Unreal structures with high intensity can be seen in the area of 30 Dor $\left(05 \mathrm{~h} 38 \mathrm{~m} 40 \mathrm{~s},-69^{\circ} 06^{\prime} 09^{\prime \prime}\right)$. At the top right corner strong sidelobes are produced by a high intensity source outside the snapshot field

We used the highest of these values to determine the completeness level, $C$, above which the sources in the field are accepted as real. These completeness levels are listed in Table 1 uncorrected for primary beam attenuation. Most fields are complete to about 6 mJy peak flux density at $1.4 \mathrm{GHz}$ and to about $3 \mathrm{mJy}$ at $2.4 \mathrm{GHz}$. In field 2D6 confusion by the bright extended emission of SNR 0525-696 makes source finding for this area impossible.

\section{The source list}

The positions, peak flux densities, $S_{\mathrm{p}}$, and integrated flux densities, $S_{\mathrm{i}}$, of the sources were determined using the AIPS routine IMFIT. The integrated flux density of a source is given by

$S_{\mathrm{i}}=S_{\mathrm{p}} \cdot \frac{\Theta_{S \max } \cdot \Theta_{S \min }}{\Theta_{B \max } \cdot \Theta_{B \min }}$

where $\Theta_{S \max }$ and $\Theta_{S \min }$ are the major and minor axis of the source response as fitted by a Gaussian to the map and $\Theta_{B \max }$ and $\Theta_{B \min }$ are the gaussian major and minor axis of the synthesized beam. The peak flux densities are much better determined than the integrated flux densities, because of the missing short spacings on the $u v$ plane. 
Table 3 lists all sources found in the survey at $1.4 \mathrm{GHz}$ that fulfill the criteria described in Sect. 2.3. A sample of 113 sources is found. The source name is given in the first column. It is derived from the standard IAU system of source nomenclature (Lortet et al. 1994), namely MDM NNN (e.g. MDM 114). The letters "NNN" indicate the sequence number within the catalogue. The position of the source is listed in the next two columns. If a source has been detected in two different fields, we use the one with the closer field centre. The integral and peak flux densities are given in Cols. 4 and 5. Both of these values have been corrected for the primary beam attenuation. Columns 6 and 7 show the spacings used and the value of the tapering when imaging the data with MX. Column 8 gives the field in which the source is detected.

The maps at $2.4 \mathrm{GHz}$ show a better quality than the data at $1.4 \mathrm{GHz}$. This is due to the smaller amplitude of the extended emission at the shorter wavelength. 70 point sources have been detected at $2.4 \mathrm{GHz}$; these are listed in Table 4. Source MDM 6 has not been detected at $1.4 \mathrm{GHz}$ due to the less sensitivity at this frequency. The source positions at $1.4 \mathrm{GHz}$ and $2.4 \mathrm{GHz}$ agree very well. The position difference is mainly below $2^{\prime \prime}$ and independent of the flux density of the source. For 69 sources the spectral index $\alpha$, with $S_{\mathrm{p}}(\nu) \sim \nu^{\alpha}$, between 1.4 and $2.4 \mathrm{GHz}$ has been estimated using the peak flux density $S_{\mathrm{p}}$. The values are listed in Col. 9. The mean spectral index for all 69 sources is $\langle\alpha\rangle=-0.72 \pm 0.09$.

\subsection{Missing flux density}

After filtering out the extended structures, only compact objects and the cores of extended sources appear in the final images. Excluding baselines below $3 k \lambda$ the survey is only sensitive to structures smaller than about $54^{\prime \prime}$ at $1.4 \mathrm{GHz}$ and $34^{\prime \prime}$ at $2.4 \mathrm{GHz}$. Assuming a distance of $50 \mathrm{kpc}$ for the LMC (Westerlund 1992), these values correspond to sizes smaller than $13 \mathrm{pc}(1.4 \mathrm{GHz})$ and $8 \mathrm{pc}$ $(2.4 \mathrm{GHz})$. To illustrate this effect for several of our snapshot fields we added artificial $100 \mathrm{mJy}$ sources of different sizes to the $u v$-data using the task UVSUB and then processed the data with MX in the same way that we had processed the field before. After mapping and cleaning the field with MX, flux is lost for the artificial source depending on its size. Figure 2 shows the distribution of the integral flux densities for artificial sources of different angular sizes. For a diameter of about $3^{\prime \prime}$, we lose $10 \%$ of the flux. This loss of flux depends on the $u v$-coverage of the field. For fields where only baselines above $6 k \lambda$ have been used because of very extended emission, much more flux is lost. If a $100 \mathrm{mJy}$ source with an angular size of $3^{\prime \prime}$ is added into field 5D6, the missing flux is on the order of $19 \%$. For angular sizes above $10^{\prime \prime}$, the flux drops off quite sharply. This implies that the flux densities of the snapshot sources are lower limits in the case of resolved sources.

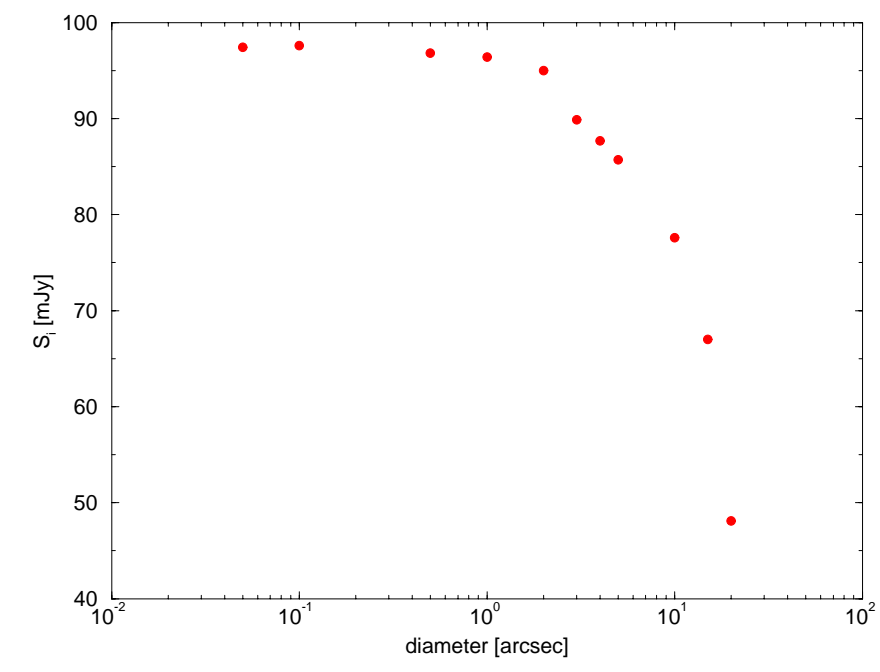

Fig. 2. Integrated flux densities for simulated $100 \mathrm{mJy}$ sources of several sizes

\section{Identifications}

\subsection{Comparison with radio observations}

We compared our data with several single dish surveys of the LMC, the Molonglo observations at $408 \mathrm{MHz}$ (Clarke et al. 1976) and the Parkes observations at $5 \mathrm{GHz}$ (McGee et al. 1972a) and $4.75 \mathrm{GHz}$ (Filipovic et al. 1995).

We identify sources of the ATCA catalogue with objects listed in the Molonglo Radio Source Catalogue 4, if the difference in position is smaller than the HPBW of the Molonglo telescope (2'.6). Nearly all sources above $50 \mathrm{mJy}$ can be identified with an MC4 object (see Table 3 Col. 9). To compare the peak flux densities of both surveys, we estimated the corresponding flux density of the MC4 source at $1.4 \mathrm{GHz}$ by using our spectral indices between 1.4 and $2.4 \mathrm{GHz}$ (Table 4 Col. 9). There are 21 usable objects for this comparison. Most of the estimated flux densities show a much higher value compared with the measured fluxes of the ATCA sources. This discrepancy may be accounted for solely by flux loss in the Compact Array observations of extended sources as compared with the filled aperture telescope. Furthermore many compact extragalactic objects are known to be variable. However, there may also be a component of this discrepancy arising from bias in the quoted spectral indices because of the different amount of the flux loss at 1.4 and $2.4 \mathrm{GHz}$. Only a few sources show similar flux densities in both surveys. These objects (MDM 1, MDM 64, MDM 84, MDM 90, MDM 100) seem to be point sources. Due to the high resolution of the ATCA, the strong source MC4(0524-708) was separated into two components.

There are only 10 sources which can be identified with objects found by McGee et al. (1972a) at $6 \mathrm{~cm}$ (Table 3 Col. 9), if we demand that the position difference from the ATCA object to the one of the MC-catalogue is smaller 
Table 3. The source list at $1.4 \mathrm{GHz}$

\begin{tabular}{|c|c|c|c|c|c|c|c|c|}
\hline Source name & $\begin{array}{l}\text { R.A. (J2000) } \\
{\left[\begin{array}{lll}\text { h } & \text { s }]\end{array}\right.}\end{array}$ & $\begin{array}{l}\text { Dec. }(\mathrm{J} 2000) \\
{\left[{ }^{0},{ }^{\prime}\right.}\end{array}$ & $\begin{array}{r}S_{\mathrm{i}} \\
{[\mathrm{mJy}]}\end{array}$ & $\begin{array}{r}S_{\mathrm{p}} \\
{[\mathrm{mJy}]}\end{array}$ & $\begin{array}{l}u v \mathrm{r} \\
{[k \lambda]}\end{array}$ & $\begin{array}{l}u v \mathrm{t} \\
{[k \lambda]}\end{array}$ & field & Identifications \\
\hline MDM 1 & 051715.95 & -702358.9 & 80 & 70 & $>3$ & 15 & 1D7 & MC4(0517-704), LMC B0517-7026 \\
\hline MDM 2 & 051802.58 & -675542.3 & 52 & 36 & $>3$ & 15 & $1 \mathrm{D} 2$ & MC4(0518-679), LMC B0518-6759, DEM129 \\
\hline MDM 3 & 051832.74 & -693522.8 & 326 & 288 & $>5$ & 15 & 1D6 & MC4(0518-696A), LMC B0519-6941 \\
\hline MDM 4 & 051850.08 & -690933.6 & 22 & 23 & $>3$ & 15 & 1D5 & MC4(0519-691), LMC B0519-6916, DEM132b \\
\hline MDM 5 & 051855.39 & -690900.9 & 6 & 7 & $>3$ & 15 & 1D5 & MC4(0519-691), DEM132b \\
\hline MDM 7 & 051916.42 & $-7014 \quad 16.1$ & 19 & 19 & $>3$ & 15 & 1D7 & \\
\hline MDM 8 & 051925.72 & $\begin{array}{llll}-67 & 47 & 02.1\end{array}$ & 15 & 17 & $>3$ & 15 & 1D2 & \\
\hline MDM 9 & 052007.00 & -680224.4 & 64 & 32 & $>5$ & 15 & 1D3 & MC4(0520-680), LMC B0520-6804 \\
\hline MDM 10 & 052019.27 & $\begin{array}{lll}-68 & 12 & 43.1\end{array}$ & 14 & 13 & $>5$ & 15 & 1D3 & \\
\hline MDM 11 & 052026.09 & -704251.5 & 8 & 9 & $>3$ & 15 & 1D8 & \\
\hline MDM 12 & 052105.24 & -695941.2 & 111 & 104 & $>5$ & 15 & 1D6 & MC4(0521-700), LMC B0521-7002 \\
\hline MDM 13 & 052108.56 & -674331.0 & 30 & 28 & $>3$ & 15 & 1D2 & DEM151 \\
\hline MDM 14 & 052118.09 & $\begin{array}{lll}-71 & 02 & 20.1\end{array}$ & 28 & 24 & $>3$ & 15 & $1 \mathrm{D} 8$ & \\
\hline MDM 15 & 052127.58 & -670724.1 & 36 & 35 & $>3$ & 15 & $1 \mathrm{D} 1$ & LMC B0521-6710 \\
\hline MDM 16 & 052145.09 & $\begin{array}{lll}-70 & 20 & 48.8\end{array}$ & 5 & 5 & $>3$ & 15 & 1D7 & \\
\hline MDM 17 & 052217.40 & -672740.4 & 36 & 27 & $>3$ & 15 & 1D1 & \\
\hline MDM 18 & 052229.52 & -703756.8 & 148 & 135 & $>3$ & 15 & $1 \mathrm{D} 8$ & MC4(0523-706), LMC B0523-7040 \\
\hline MDM 19 & 052256.13 & -690408.5 & 16 & 19 & $>5$ & 15 & 2D5 & DEM177 \\
\hline MDM 20 & 052258.87 & -684429.3 & 23 & 22 & $>3$ & 15 & $2 \mathrm{D} 4$ & \\
\hline MDM 21 & 052340.94 & -705020.7 & 225 & 210 & $>3$ & 15 & $2 \mathrm{D} 8$ & MC4(0524-708), MC37, LMC B0524-7053 \\
\hline MDM 22 & 052340.97 & -705122.3 & 245 & 220 & $>3$ & 15 & $2 \mathrm{D} 8$ & MC4(0524-708), MC37, LMC B0524-7053 \\
\hline MDM 23 & $05 \quad 2505.71$ & -682818.5 & 13 & 9 & $>3$ & 15 & 2D3 & LMC B0525-6831, DEM180 \\
\hline MDM 24 & $05 \quad 2505.74$ & -683700.9 & 9 & 9 & $>3$ & 15 & $2 \mathrm{D} 4$ & \\
\hline MDM 25 & $05 \quad 25 \quad 17.22$ & -672247.4 & 62 & 58 & $>3$ & 15 & 2D1 & DEM192 \\
\hline MDM 26 & 052529.95 & -673130.5 & 14 & 10 & $>3$ & 15 & $2 \mathrm{D} 1$ & LMC B0526-6731, DEM192 \\
\hline MDM 27 & 052533.69 & $\begin{array}{lll}-68 & 41 & 21.2\end{array}$ & 8 & 10 & $>3$ & 15 & $2 \mathrm{D} 4$ & \\
\hline MDM 28 & 052611.85 & -702246.8 & 14 & 14 & $>3$ & 15 & 2D7 & \\
\hline MDM 29 & 052624.43 & $\begin{array}{lll}-68 & 15 & 11.2\end{array}$ & 43 & 26 & $>3$ & 15 & $2 \mathrm{D} 3$ & DEM203 \\
\hline MDM 30 & 052635.48 & -674909.3 & 95 & 89 & $>3$ & 15 & $2 \mathrm{D} 2$ & LMC B0526-6751 \\
\hline MDM 31 & 052745.92 & -675927.1 & 35 & 37 & $>3$ & 15 & $2 \mathrm{D} 2$ & \\
\hline MDM 32 & $05 \quad 2749.22$ & -703644.0 & 54 & 50 & $>3$ & 15 & $2 \mathrm{D} 8$ & MC4(0528-706), LMC B0528-7038, DEM208 \\
\hline MDM 33 & 052754.56 & -691140.7 & 15 & 13 & $>5$ & 15 & 2D5 & LMC B0528-6914, DEM210 \\
\hline MDM 34 & 052800.03 & -702541.5 & 22 & 20 & $>3$ & 15 & 3D7 & \\
\hline MDM 35 & 052847.68 & -683623.1 & 22 & 21 & $>3$ & 15 & $3 \mathrm{D} 4$ & \\
\hline MDM 36 & 052947.89 & $\begin{array}{lll}-69 & 02 & 11.7\end{array}$ & 15 & 14 & $>3$ & 15 & 3D5 & \\
\hline MDM 37 & 052950.59 & $\begin{array}{lll}-67 & 01 & 14.7\end{array}$ & 14 & 11 & $>3$ & 15 & 3D1 & LMC B0529-6702, DEM214 \\
\hline MDM 38 & 052951.64 & -674934.5 & 188 & 150 & $>3$ & 15 & $3 \mathrm{D} 2$ & MC4(0529-678), LMC B0529-6752 \\
\hline MDM 39 & 052951.83 & $\begin{array}{lll}-68 & 10 & 18.4\end{array}$ & 6 & 6 & $>3$ & 15 & 3D3 & LMC B0530-6814 \\
\hline MDM 40 & 053122.47 & -701154.6 & 11 & 12 & $>3$ & 15 & $3 \mathrm{D} 7$ & LMC B0531-7010 \\
\hline MDM 41 & 053142.57 & -683453.9 & 7 & 8 & $>3$ & 15 & $3 \mathrm{D} 4$ & LMC B0532-6833 \\
\hline MDM 42 & 053143.94 & -704925.8 & 18 & 18 & $>3$ & 15 & $3 \mathrm{D} 8$ & DEM221 \\
\hline MDM 43 & 053154.71 & -682639.0 & 6 & 7 & $>3$ & 15 & 3D3 & LMC B0532-6833 \\
\hline MDM 44 & 053207.16 & -675414.2 & 34 & 34 & $>3$ & 15 & 3D2 & \\
\hline MDM 45 & 053209.65 & -684308.1 & 11 & 11 & $>3$ & 15 & 3D4 & MC55, LMC B0532-6841, DEM226 \\
\hline MDM 46 & 053211.06 & -683024.4 & 9 & 9 & $>3$ & 15 & 3D3 & MC56, LMC B0532-6833 \\
\hline MDM 47 & 053216.50 & -702357.3 & 10 & 10 & $>3$ & 15 & $3 \mathrm{D} 7$ & \\
\hline MDM 48 & 053244.99 & -700128.5 & 75 & 71 & $>3$ & 15 & 3D7 & LMC B0533-7001 \\
\hline MDM 49 & 053248.24 & -682359.3 & 9 & 9 & $>3$ & 15 & 3D3 & \\
\hline MDM 50 & $0532 \quad 50.32$ & $\begin{array}{llll}-68 & 20 & 12.6\end{array}$ & 11 & 12 & $>3$ & 15 & 3D3 & \\
\hline MDM 51 & 053252.80 & -694622.5 & 33 & 30 & $>3$ & 15 & 3D6 & DEM246 \\
\hline MDM 52 & $0532 \quad 53.24$ & -670944.6 & 28 & 22 & $>3$ & 15 & 3D1 & \\
\hline MDM 53 & 053254.21 & -704028.4 & 16 & 15 & $>3$ & 15 & $3 \mathrm{D} 8$ & \\
\hline MDM 54 & 053315.32 & $\begin{array}{lll}-69 & 12 & 46.8\end{array}$ & 9 & 8 & $>3$ & 15 & 3D5 & DEM232 \\
\hline MDM 55 & 053318.70 & -674000.2 & 29 & 31 & $>3$ & 15 & $3 \mathrm{D} 2$ & LMC B0532-6743, DEM231 \\
\hline MDM 56 & 053342.44 & $\begin{array}{lll}-68 & 46 & 03.3\end{array}$ & 50 & 32 & $>3$ & 15 & $3 \mathrm{D} 4$ & LMC B0534-6847, DEM233 \\
\hline MDM 57 & 053408.28 & -703717.4 & 9 & 11 & $>3$ & 15 & $4 \mathrm{D} 8$ & \\
\hline MDM 58 & 053448.37 & -675600.1 & 46 & 39 & $>3$ & 15 & $4 \mathrm{D} 2$ & MC4(0534-679) \\
\hline MDM 59 & 053524.70 & -673509.6 & 23 & 11 & $>3$ & 15 & $4 \mathrm{D} 2$ & $\begin{array}{l}\text { MC4(0535-676A), MC64, LMC B0535-6736, } \\
\text { DEM241 }\end{array}$ \\
\hline MDM 60 & 053526.51 & -671704.5 & 67 & 56 & $>3$ & 15 & $4 \mathrm{D} 1$ & MC4(0535-673), LMC B0535-6718 \\
\hline MDM 61 & 053537.48 & -685508.0 & 63 & 60 & $>6$ & 0 & $4 \mathrm{D} 4$ & LMC B0535-6857 \\
\hline MDM 62 & 053605.06 & -691845.9 & 78 & 84 & $>6$ & 0 & $4 \mathrm{D} 5$ & DEM263 \\
\hline MDM 63 & 053623.89 & -702543.0 & 9 & 11 & $>3$ & 15 & $4 \mathrm{D} 7$ & \\
\hline MDM 64 & 053636.42 & -670736.1 & 65 & 62 & $>3$ & 15 & $4 \mathrm{D} 1$ & MC4(0536-671), DEM257 \\
\hline MDM 65 & 053657.17 & -69 1329.0 & 174 & 149 & $>6$ & 0 & 4D5 & $\begin{array}{l}\text { MC4(0537-692A), LMC B0538-6911, } \\
\text { DEM263 }\end{array}$ \\
\hline MDM 66 & 053736.93 & $\begin{array}{lll}-68 & 00 & 34.3\end{array}$ & 35 & 33 & $>3$ & 15 & 4D3 & DEM268 \\
\hline MDM 67 & 053756.50 & -703514.8 & 9 & 11 & $>3$ & 15 & $4 \mathrm{D} 8$ & \\
\hline MDM 68 & 053804.78 & -695337.5 & 69 & 62 & $>6$ & 0 & $4 \mathrm{D} 6$ & LMC B0538-6956 \\
\hline MDM 69 & 053809.01 & $\begin{array}{lll}-70 & 49 & 32.1\end{array}$ & 10 & 11 & $>3$ & 15 & $4 \mathrm{D} 8$ & \\
\hline MDM 70 & 053816.15 & -705636.4 & 21 & 20 & $>3$ & 15 & $4 \mathrm{D} 8$ & \\
\hline MDM 71 & 053821.43 & -704109.3 & 37 & 22 & $>3$ & 15 & $5 \mathrm{D} 8$ & LMC B0538-7042, DEM265 \\
\hline MDM 72 & 053844.46 & -690439.2 & 75 & 56 & $>6$ & 0 & 5D5 & $\begin{array}{l}\text { MC4(0539-691), MC74, LMC B0539-6907, } \\
\text { DEM263 }\end{array}$ \\
\hline MDM 73 & 053929.92 & -700328.9 & 24 & 22 & $>3$ & 15 & $4 \mathrm{D} 7$ & \\
\hline
\end{tabular}


Table 3. continued

\begin{tabular}{|c|c|c|c|c|c|c|c|c|}
\hline Source name & $\begin{array}{l}\text { R.A. (J2000) } \\
{[\text { h } \mathrm{m} \text { s] }}\end{array}$ & $\begin{array}{l}\text { Dec. }(\mathrm{J} 2000) \\
{\left[\begin{array}{lll}0 & 1 & \prime \prime\end{array}\right]}\end{array}$ & $\begin{array}{r}S_{\mathrm{i}} \\
{[\mathrm{mJy}]}\end{array}$ & $\begin{array}{r}S_{\mathrm{p}} \\
{[\mathrm{mJy}]}\end{array}$ & $\begin{array}{l}u v \mathrm{r} \\
{[k \lambda]}\end{array}$ & $\begin{array}{l}u v \mathrm{t} \\
{[k \lambda]}\end{array}$ & field & Identifications \\
\hline MDM 74 & 053937.56 & -694527.1 & 50 & 41 & $>6$ & 0 & 5D6 & MC77, LMC B0540-6946, DEM271 \\
\hline MDM 75 & 053945.82 & -693838.8 & 33 & 31 & $>6$ & 0 & $5 \mathrm{D} 6$ & MC4(0540-696B), MC76, DEM284 \\
\hline MDM 76 & 053954.90 & -700745.8 & 12 & 12 & $>3$ & 15 & $4 \mathrm{D} 7$ & \\
\hline MDM 77 & 054002.05 & -690619.8 & 24 & 27 & $>6$ & 0 & 5D5 & DEM263 \\
\hline MDM 78 & 054004.65 & -694439.6 & 23 & 21 & $>6$ & 0 & $5 \mathrm{D} 6$ & $\begin{array}{l}\text { MC4(0540-697A), MC77, LMC B0540-6946, } \\
\text { DEM271 }\end{array}$ \\
\hline MDM 79 & 054010.39 & -671814.8 & 75 & 70 & $>3$ & 15 & $5 \mathrm{D} 1$ & LMC B0540-6719 \\
\hline MDM 80 & $05 \quad 4011.43$ & -691955.5 & 40 & 35 & $>6$ & 0 & 5D5 & $\begin{array}{l}\text { MC4(0540-693), MC78, LMC B0540-6921, } \\
\text { DEM269 }\end{array}$ \\
\hline MDM 81 & $0541 \quad 16.68$ & -675955.5 & 21 & 16 & $>3$ & 15 & $5 \mathrm{D} 2$ & MC4(0541-680), LMC B0541-6801 \\
\hline MDM 82 & $05 \quad 4127.23$ & -673952.1 & 44 & 34 & $>3$ & 15 & $5 \mathrm{D} 2$ & \\
\hline MDM 83 & 054131.58 & -704629.4 & 20 & 19 & $>3$ & 15 & $5 \mathrm{D} 8$ & \\
\hline MDM 84 & 054133.03 & -670616.6 & 69 & 62 & $>3$ & 15 & $5 \mathrm{D} 1$ & MC4(0541-671), LMC B0541-6707 \\
\hline MDM 85 & 054159.09 & -681543.4 & 30 & 31 & $>3$ & 15 & 5D3 & \\
\hline MDM 86 & 054244.53 & -672554.4 & 16 & 14 & $>3$ & 15 & $5 \mathrm{D} 1$ & LMC B0543-6730 \\
\hline MDM 87 & 054256.33 & -701736.4 & 12 & 11 & $>5$ & 15 & $5 \mathrm{D} 7$ & \\
\hline MDM 88 & $05 \quad 4306.72$ & $\begin{array}{llll}-71 & 04 & 05.7\end{array}$ & 58 & 43 & $>3$ & 15 & $5 \mathrm{D} 8$ & MC4(0543-710), LMC B0543-7105 \\
\hline MDM 89 & 054314.49 & -684436.1 & 74 & 70 & $>3$ & 15 & $5 \mathrm{D} 4$ & \\
\hline MDM 90 & $\begin{array}{lll}05 & 43 & 15.29\end{array}$ & -680653.9 & 128 & 109 & $>3$ & 15 & $5 \mathrm{D} 3$ & MC4(0543-681), LMC B0543-6808 \\
\hline MDM 91 & $05 \quad 43 \quad 17.17$ & -671509.8 & 7 & 7 & $>3$ & 15 & $5 \mathrm{D} 1$ & \\
\hline MDM 92 & 054356.08 & $\begin{array}{lll}-68 & 17 & 32.4\end{array}$ & 13 & 12 & $>3$ & 15 & 5D3 & \\
\hline MDM 93 & $05 \quad 4504.54$ & -693929.7 & 18 & 14 & $>5$ & 0 & $6 \mathrm{D} 6$ & DEM310 \\
\hline MDM 94 & 054527.65 & -694624.4 & 9 & 7 & $>5$ & 0 & 6D6 & LMC B0545-6947, DEM311 \\
\hline MDM 95 & 054550.52 & -684557.8 & 62 & 53 & $>3$ & 15 & $6 \mathrm{D} 4$ & MC4(0546-687) \\
\hline MDM 96 & $\begin{array}{lll}05 & 46 & 12.16\end{array}$ & $\begin{array}{lll}-68 & 00 & 15.9\end{array}$ & 21 & 21 & $>3$ & 15 & $6 \mathrm{D} 3$ & LMC B0546-6802 \\
\hline MDM 97 & 054623.49 & -665548.2 & 16 & 13 & $>3$ & 15 & $6 \mathrm{D} 1$ & \\
\hline MDM 98 & 054629.15 & $\begin{array}{lll}-70 & 26 & 40.7\end{array}$ & 83 & 50 & $>5$ & 15 & $6 \mathrm{D} 7$ & MC4(0547-704) \\
\hline MDM 99 & 054652.85 & -683248.7 & 13 & 12 & $>3$ & 15 & $6 \mathrm{D} 3$ & \\
\hline MDM 100 & 054745.37 & $\begin{array}{lll}-67 & 45 & 07.1\end{array}$ & 90 & 83 & $>3$ & 15 & $6 \mathrm{D} 2$ & MC4(0547-677), LMC B0547-6746 \\
\hline MDM 101 & 054750.31 & -672802.5 & 6 & 6 & $>3$ & 15 & $6 \mathrm{D} 1$ & LMC B0547-6729 \\
\hline MDM 102 & $05 \quad 4751.41$ & -694543.5 & 7 & 8 & $>5$ & 0 & 6D6 & LMC B0547-6942 \\
\hline MDM 103 & 054757.70 & $\begin{array}{lll}-70 & 20 & 16.1\end{array}$ & 12 & 12 & $>5$ & 15 & $6 \mathrm{D} 7$ & LMC B0548-7025 \\
\hline MDM 104 & $0548 \quad 45.46$ & -665726.7 & 13 & 8 & $>3$ & 15 & $6 \mathrm{D} 1$ & \\
\hline MDM 105 & 054855.14 & -703926.7 & 21 & 21 & $>3$ & 15 & $6 \mathrm{D} 8$ & LMC B0549-7037 \\
\hline MDM 106 & 054939.70 & -693823.6 & 14 & 14 & $>5$ & 0 & $6 \mathrm{D} 6$ & \\
\hline MDM 107 & 055015.89 & -673603.5 & 24 & 21 & $>3$ & 15 & $6 \mathrm{D} 2$ & \\
\hline MDM 108 & 055032.90 & -682055.5 & 48 & 34 & $>3$ & 15 & $6 \mathrm{D} 3$ & MC4(0550-683), LMC B0550-6823 \\
\hline MDM 109 & 055048.97 & -703434.7 & 47 & 28 & $>3$ & 15 & $6 \mathrm{D} 8$ & \\
\hline MDM 110 & 055130.31 & -691632.8 & 91 & 79 & $>3$ & 15 & 6D5 & MC4(0551-692), LMC B0551-6917 \\
\hline MDM 111 & 055139.49 & $-6843 \quad 17.3$ & 30 & 30 & $>3$ & 15 & $6 \mathrm{D} 4$ & MC4(0552-687), LMC B0552-6843 \\
\hline MDM 112 & $05 \quad 5205.81$ & -681441.9 & 178 & 153 & $>3$ & 15 & 6D3 & MC4(0552-682), LMC B0552-6815 \\
\hline MDM 113 & 055227.83 & -694802.9 & 75 & 45 & $>5$ & 0 & $6 \mathrm{D} 6$ & MC4(0552-698), LMC B0552-6948 \\
\hline MDM 114 & 055228.46 & -702137.0 & 56 & 44 & $>5$ & 15 & $6 \mathrm{D} 7$ & MC4(0552-703), LMC B0552-7022 \\
\hline
\end{tabular}

than the HPBW of the Parkes telescope, which is about $4^{\prime}$ at $6 \mathrm{~cm}$. The flux densities of all sources observed with the single dish telescope are much higher than those of the compact ATCA sources.

We identified a compact source in the snapshot field of $3^{\circ} \times 4^{\circ}$ with an object observed with Parkes at $4.75 \mathrm{GHz}$ (Filipovic et al. 1995), if the difference in position is smaller than the HPBW of 4.8 (Table 3 Col. 9 abbreviation LMC). For all 53 identified sources the flux density of the Parkes object is higher than the value of the ATCA source at $1.4 \mathrm{GHz}$. There is also a large position difference for most objects.

The poor correspondance between compact sources detected with the ATCA and the Parkes sources is common also in galactic plane surveys (Garwood et al. 1988), and arises from the fact that most of the continuum flux from a galaxy disk comes from structures much larger than a few parsecs, which is our effective resolution at the distance of the LMC.
From the comparison of our snapshot sources with radio objects from single dish observations, we conclude that most of our discrete objects are compact cores of extended sources or background objects.

\subsection{Identification with $H \alpha$ emitting objects}

Comparison of the radio point source positions with $\mathrm{H} \alpha$ emitting objects helps us to find sources intrinsic to the LMC. Figure 3 shows the distribution of the compact radio sources on the $\mathrm{H} \alpha$ map of Kennicutt et al. (1995).

A compact radio source is regarded as being identified with an $\mathrm{H} \alpha$ object of the Davies-Elliot-Meaburn catalogue (DEM), if the radio source lies within the extent of the DEM object. We find 32 radio point sources associated with an $\mathrm{H} \alpha$ emitting object. These might be compact HII regions or young SNRs. Such objects can show high brightness knots embedded in the extended DEM object. $\mathrm{H} \alpha$ observations at 
Table 4. The source list at $2.4 \mathrm{GHz}$

\begin{tabular}{|c|c|c|c|c|c|c|c|c|}
\hline Source name & $\begin{array}{l}\text { R.A. (J2000) } \\
{\left[\begin{array}{lll}\text { h } & \text { m }\end{array}\right]}\end{array}$ & $\begin{array}{l}\text { Dec. (J2000) } \\
{\left[\begin{array}{lll}0 & 1 & \prime \prime\end{array}\right]}\end{array}$ & $\begin{array}{r}S_{\mathrm{i}} \\
{[\mathrm{mJy}]}\end{array}$ & $\begin{array}{r}S_{\mathrm{p}} \\
{[\mathrm{mJy}]}\end{array}$ & $\begin{array}{l}u v \mathrm{r} \\
{[k \lambda]}\end{array}$ & $\begin{array}{l}u v \mathrm{t} \\
{[k \lambda]}\end{array}$ & field & $\alpha$ \\
\hline MDM 1 & $\begin{array}{llll}05 & 17 & 16.07\end{array}$ & -702359.0 & 61 & 49 & $>2$ & 30 & 1D7 & -0.67 \\
\hline MDM 2 & $\begin{array}{lll}05 & 18 & 02.61\end{array}$ & -675543.3 & 19 & 16 & $>3$ & 30 & 1D2 & -1.48 \\
\hline MDM 3 & $\begin{array}{llll}0 & 18 & 32.72\end{array}$ & -693522.5 & 259 & 219 & $>3$ & 30 & 1D6 & -0.50 \\
\hline MDM 4 & $\begin{array}{lll}05 & 18 & 50.03\end{array}$ & $\begin{array}{llll}-69 & 09 & 32.0\end{array}$ & 6 & 6 & $>3$ & 30 & 1D5 & -2.55 \\
\hline MDM 5 & $05 \quad 18 \quad 55.25$ & -690900.7 & 5 & 5 & $>3$ & 30 & 1D5 & -0.57 \\
\hline MDM 6 & $\begin{array}{lll}05 & 19 & 10.74\end{array}$ & $-68 \quad 1544.4$ & 6 & 6 & $>3$ & 30 & 1D3 & \\
\hline MDM 7 & $\begin{array}{lll}05 & 19 & 16.38\end{array}$ & -701416.5 & 29 & 20 & $>2$ & 30 & 1D7 & +0.05 \\
\hline MDM 8 & $\begin{array}{lll}05 & 19 & 25.77\end{array}$ & -674701.6 & 11 & 11 & $>3$ & 30 & 1D2 & -0.79 \\
\hline MDM 9 & $\begin{array}{llll}05 & 20 & 07.74\end{array}$ & $-6802 \quad 25.0$ & 24 & 20 & $>3$ & 30 & 1D3 & -0.87 \\
\hline MDM 10 & $\begin{array}{lll}05 & 20 & 19.04\end{array}$ & $\begin{array}{lll}-68 & 12 & 42.7\end{array}$ & 6 & 5 & $>3$ & 30 & 1D3 & -1.69 \\
\hline MDM 11 & $05 \quad 2025.85$ & -704252.5 & 7 & 8 & $>3$ & 30 & $1 \mathrm{D} 8$ & -0.28 \\
\hline MDM 12 & $\begin{array}{lll}05 & 21 & 05.24\end{array}$ & -695941.2 & 91 & 92 & $>3$ & 30 & 1D6 & -0.22 \\
\hline MDM 13 & $\begin{array}{lll}05 & 21 & 08.61\end{array}$ & -674331.4 & 18 & 14 & $>3$ & 30 & $1 \mathrm{D} 2$ & -1.27 \\
\hline MDM 15 & 052127.67 & -670724.3 & 33 & 30 & $>3$ & 30 & 1D1 & -0.30 \\
\hline MDM 18 & $05 \quad 2229.55$ & -703756.6 & 68 & 68 & $>3$ & 30 & $1 \mathrm{D} 8$ & -1.28 \\
\hline MDM 21 & 052340.86 & -705020.3 & 144 & 120 & $>3$ & 30 & $2 \mathrm{D} 8$ & -1.03 \\
\hline MDM 22 & $05 \quad 2341.01$ & -705123.8 & 156 & 141 & $>3$ & 30 & $2 \mathrm{D} 8$ & -0.82 \\
\hline MDM 24 & 052505.62 & $\begin{array}{llll}-68 & 37 & 00.7\end{array}$ & 8 & 8 & $>3$ & 30 & $2 \mathrm{D} 4$ & -0.23 \\
\hline MDM 25 & $\begin{array}{lll}05 & 25 & 17.22\end{array}$ & -672247.5 & 67 & 61 & $>3$ & 30 & 2D1 & +0.09 \\
\hline MDM 27 & $\begin{array}{lll}05 & 2533.87\end{array}$ & $\begin{array}{lll}-68 & 41 & 20.9\end{array}$ & 6 & 6 & $>3$ & 30 & $2 \mathrm{D} 4$ & -0.92 \\
\hline MDM 28 & $\begin{array}{lll}05 & 26 & 11.92\end{array}$ & $\begin{array}{lll}-70 & 22 & 47.4\end{array}$ & 6 & 6 & $>3$ & 30 & 2D7 & -1.57 \\
\hline MDM 29 & 052624.76 & -681514.6 & 14 & 14 & $>3$ & 30 & 2D3 & -1.16 \\
\hline MDM 30 & $05 \quad 2635.34$ & -674909.4 & 119 & 111 & $>0$ & 30 & 2D2 & +0.42 \\
\hline MDM 31 & 052745.90 & -675927.1 & 39 & 46 & $>0$ & 30 & $2 \mathrm{D} 2$ & +0.41 \\
\hline MDM 32 & $05 \quad 2749.20$ & -703644.2 & 35 & 31 & $>3$ & 30 & $2 \mathrm{D} 8$ & -0.91 \\
\hline MDM 35 & $05 \quad 2847.65$ & -683622.9 & 22 & 24 & $>3$ & 30 & $3 \mathrm{D} 4$ & +0.24 \\
\hline MDM 38 & 052951.64 & -674933.6 & 101 & 55 & $>3$ & 30 & 3D2 & -1.85 \\
\hline MDM 40 & 053122.53 & -701154.5 & 5 & 6 & $>3$ & 30 & $3 \mathrm{D} 7$ & -1.24 \\
\hline MDM 42 & 053143.93 & -704925.4 & 16 & 14 & $>3$ & 30 & $3 \mathrm{D} 8$ & -0.39 \\
\hline MDM 44 & 053207.06 & -675414.3 & 22 & 18 & $>3$ & 30 & $3 \mathrm{D} 2$ & -1.15 \\
\hline MDM 45 & 053209.69 & -684307.9 & 11 & 12 & $>3$ & 30 & $3 \mathrm{D} 4$ & +0.06 \\
\hline MDM 48 & 053244.99 & $\begin{array}{llll}-70 & 01 & 28.7\end{array}$ & 48 & 47 & $>3$ & 30 & $3 \mathrm{D} 7$ & -0.78 \\
\hline MDM 50 & 053250.28 & $\begin{array}{llll}-68 & 20 & 12.8\end{array}$ & 4 & 5 & $>3$ & 30 & 3D3 & -1.54 \\
\hline MDM 51 & $0532 \quad 52.81$ & -694622.8 & 22 & 18 & $>3$ & 30 & $3 \mathrm{D} 6$ & -0.95 \\
\hline MDM 52 & 053253.18 & -670946.1 & 19 & 18 & $>3$ & 30 & 3D1 & -0.39 \\
\hline MDM 53 & 053254.25 & -704028.3 & 25 & 25 & $>3$ & 30 & $3 \mathrm{D} 8$ & +0.95 \\
\hline MDM 55 & 053318.55 & -673959.7 & 23 & 24 & $>3$ & 30 & $3 \mathrm{D} 2$ & -0.51 \\
\hline MDM 56 & 053342.36 & -684604.0 & 18 & 9 & $>3$ & 30 & $3 \mathrm{D} 4$ & -2.33 \\
\hline MDM 60 & 053526.68 & $-67 \quad 1704.7$ & 44 & 37 & $>2$ & 30 & 4D1 & -0.76 \\
\hline MDM 61 & 053537.56 & -685508.2 & 72 & 64 & $>3$ & 30 & $4 \mathrm{D} 4$ & +0.11 \\
\hline MDM 62 & 053605.04 & $-69 \quad 1846.3$ & 49 & 46 & $>3$ & 30 & 4D5 & -1.11 \\
\hline MDM 63 & 053623.80 & -702543.6 & 9 & 10 & $>3$ & 30 & $4 \mathrm{D} 7$ & -0.26 \\
\hline MDM 64 & 053636.41 & -670736.0 & 48 & 40 & $>2$ & 30 & $4 \mathrm{D} 1$ & -0.83 \\
\hline MDM 65 & 053657.23 & -691329.0 & 116 & 110 & $>3$ & 30 & 4D5 & -0.56 \\
\hline MDM 66 & 053736.88 & $\begin{array}{llll}-68 & 00 & 33.8\end{array}$ & 14 & 12 & $>3$ & 30 & 4D3 & -1.85 \\
\hline MDM 68 & 053804.66 & $-6953 \quad 37.2$ & 29 & 33 & $>3$ & 30 & 4D6 & -1.17 \\
\hline MDM 70 & $0538 \quad 16.32$ & -705637.3 & 23 & 23 & $>3$ & 30 & $4 \mathrm{D} 8$ & +0.27 \\
\hline MDM 74 & 053937.59 & -694527.6 & 61 & 51 & $>3$ & 30 & 5D6 & +0.38 \\
\hline MDM 75 & 053945.85 & -693839.0 & 61 & 53 & $>3$ & 30 & 5D6 & +0.99 \\
\hline MDM 78 & 054004.48 & -694438.9 & 15 & 18 & $>3$ & 30 & 5D6 & -0.32 \\
\hline MDM 79 & $\begin{array}{llll}05 & 40 & 10.42\end{array}$ & $-6718 \quad 15.2$ & 74 & 63 & $>3$ & 30 & $5 \mathrm{D} 1$ & -0.18 \\
\hline MDM 80 & $0540 \quad 11.55$ & -691954.9 & 26 & 19 & $>3$ & 30 & 5D5 & -1.11 \\
\hline MDM 82 & $\begin{array}{lll}05 & 41 & 27.21\end{array}$ & -673951.2 & 18 & 17 & $>3$ & 30 & 5D2 & -1.29 \\
\hline MDM 83 & 054131.65 & -704627.9 & 11 & 13 & $>3$ & 30 & $5 \mathrm{D} 8$ & -0.70 \\
\hline MDM 84 & 054133.09 & -670616.7 & 39 & 36 & $>3$ & 30 & 5D1 & -1.03 \\
\hline MDM 85 & 054159.05 & $\begin{array}{lll}-68 & 15 & 43.1\end{array}$ & 27 & 28 & $>2$ & 30 & 5D3 & -0.21 \\
\hline MDM 86 & 054244.56 & -672554.6 & 12 & 10 & $>3$ & 30 & 5D1 & -0.57 \\
\hline MDM 87 & 054256.58 & $\begin{array}{llll}-70 & 17 & 34.7\end{array}$ & 4 & 4 & $>3$ & 30 & 5D7 & -1.85 \\
\hline MDM 89 & $0543 \quad 14.53$ & -684435.9 & 52 & 45 & $>3$ & 30 & $5 \mathrm{D} 4$ & -0.81 \\
\hline MDM 90 & $\begin{array}{lll}05 & 43 & 15.21\end{array}$ & -680653.7 & 76 & 43 & $>2$ & 30 & 5D3 & -1.71 \\
\hline MDM 91 & $05 \quad 43 \quad 17.02$ & $-67 \quad 1509.3$ & 3 & 4 & $>3$ & 30 & 5D1 & -0.97 \\
\hline MDM 94 & 054527.76 & -694622.5 & 10 & 7 & $>3$ & 30 & 6D6 & +0.04 \\
\hline MDM 95 & 054550.27 & -684557.0 & 30 & 28 & $>3$ & 30 & $6 \mathrm{D} 4$ & -1.20 \\
\hline MDM 98 & 054629.34 & -702644.3 & 30 & 30 & $>3$ & 30 & $6 \mathrm{D} 7$ & -0.95 \\
\hline MDM 100 & 054745.35 & -674507.0 & 69 & 52 & $>2$ & 30 & $6 \mathrm{D} 2$ & -0.85 \\
\hline MDM 101 & $05 \quad 4750.32$ & -672803.2 & 12 & 9 & $>3$ & 30 & 6D1 & +0.96 \\
\hline MDM 102 & 054751.27 & -694543.3 & 4 & 5 & $>3$ & 30 & 6D6 & -1.07 \\
\hline MDM 103 & 054757.45 & -702016.2 & 7 & 7 & $>3$ & 30 & $6 \mathrm{D} 7$ & -1.01 \\
\hline MDM 105 & 054855.24 & -703928.3 & 12 & 11 & $>3$ & 30 & $6 \mathrm{D} 8$ & -1.15 \\
\hline MDM 106 & $\begin{array}{llll}05 & 49 & 39.71\end{array}$ & -693823.9 & 10 & 10 & $>3$ & 30 & 6D6 & -0.62 \\
\hline
\end{tabular}




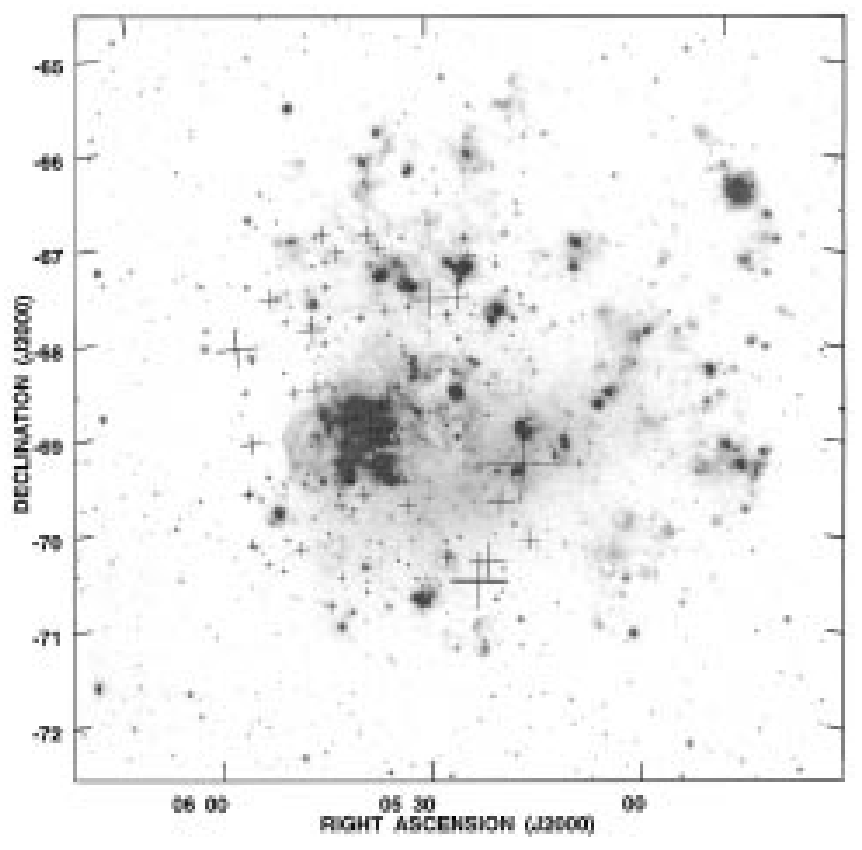

Fig. 3. Distribution of the compact radio sources on the $\mathrm{H} \alpha$ image of the LMC. The size of the crosses is proportional to the peak flux density of the source

high angular resolution performed with the aim at finding out which of the radio sources correspond to an optical emission knot, and which are background sources behind the DEM object, will be presented in a subsequent paper (Marx et al., in preparation).

\section{Source counts}

The ATCA source list of compact objects can be used to find the sky density of sources at $1.4 \mathrm{GHz}$ in the direction of the LMC. Comparing the source counts with extragalactic source count results allows us to check for incompleteness of the snapshot survey and can be useful in determining the fraction of sources intrinsic to the LMC, which should appear as an excess of counts compared with the extragalactic ones. For this comparison the integrated flux density has to be used, since the peak flux depends on the telescope beam size. The differential source counts (number of sources with a given flux density) of the LMC snapshot survey have been calculated following the method described by Condon \& Condon (1982) for their $1.411 \mathrm{GHz}$ VLA snapshot survey.

The ATCA snapshot survey is complete above the uncorrected peak flux density limits given in Table 1. Corresponding limits to the primary beam corrected peak flux densities vary with location in each map, so that the solid angle $\Omega$ in which a source of given peak flux $S_{\mathrm{p}}$ can be detected increases with $S_{\mathrm{p}}$. Each source counted must therefore be weighted by $\Omega^{-1}$ to give its proper contribution to the areal density of sources. For the calculation of
$\Omega\left(S_{\mathrm{p}}\right)$ the overlapping of the fields has been taken into account. The total counting area is $3.610^{-3} \mathrm{sr}$. The function $\Omega\left(S_{\mathrm{p}}\right)$ is plotted in Fig. 4 .

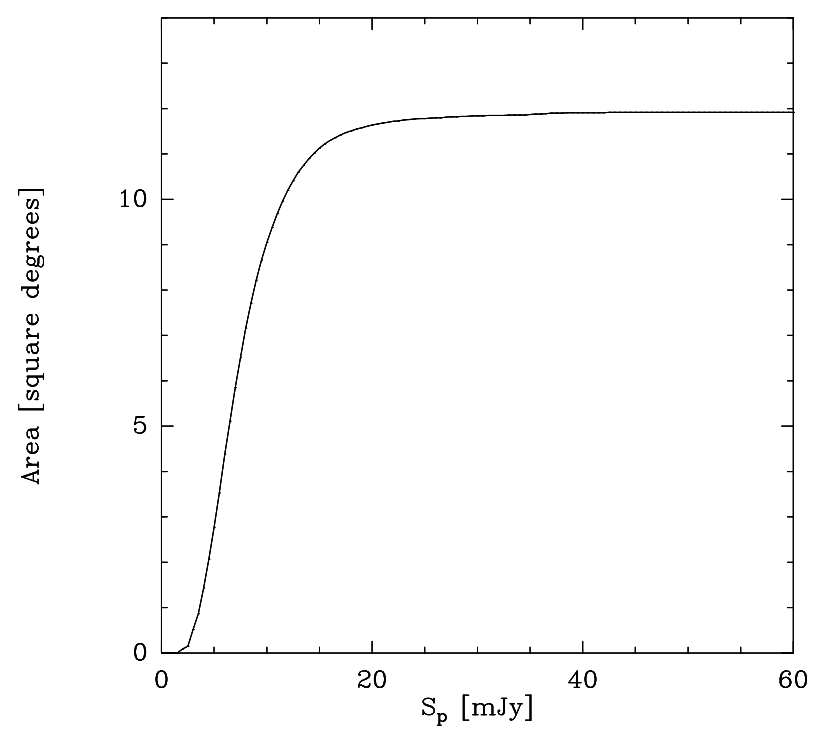

Fig. 4. Solid angle $\Omega$ in which a source with a given peak flux density has been counted

Since we want to find the sky number density of sources as a function of their integrated flux densities, we must allow for sources which are missing from the survey because they are extended. Such sources have peak flux densities, $S_{\mathrm{p}}$, below the completeness limit and integrated flux densities, $S_{\mathrm{i}}$, above this limit. We distinguish the continuum sources which are significantly resolved from those which are not by using the method of Willis et al. (1976). An observed source is defined as extended if

$R \geq 1+2 \cdot \frac{2.47 \cdot \sigma}{S_{\mathrm{p}}}$

$R$ is the Gaussian fitted area divided by that of the antenna pattern $\left(R=\frac{\Theta_{S \max } \cdot \Theta_{S \min }}{\Theta_{B \max } \cdot \Theta_{B \min }}\right)$ and $\sigma$ is the rms noise in the area of the source after primary beam correction. Assuming that the angular-size distribution of sources as faint as $4 \mathrm{mJy}$ is not significantly different from the angular-size distribution of sources in the range $35 \mathrm{mJy} \leq S_{\mathrm{i}}<140 \mathrm{mJy}$ (Downes et al. 1981), then the fraction of extended sources (0.27) in this flux density interval can be used to estimate the fraction of faint extended sources missing from the ATCA survey.

The source counts including all observed sources are listed in Table 5. The flux density interval (Col. 2) around $S_{\mathrm{c}}$ (Col. 1$)$ is defined as $2^{-1 / 2} S_{\mathrm{c}} \leq S_{\mathrm{i}}<2^{1 / 2} S_{\mathrm{c}}$. The number of resolved sources $N_{\text {res }}$ and the number of unresolved sources $N_{\text {unres }}$ found in each flux-density interval is shown in Cols. 3 and 4. The fraction of unresolved sources $f_{\text {unres }}$ is listed in Col. 5. The mean fraction of unresolved sources 
in the flux density range $35 \mathrm{mJy} \leq S_{\mathrm{i}}<140 \mathrm{mJy}$ is 0.27 , so the correction factor $K$ (Col. 6) is the fraction of unresolved sources in each flux density interval divided by 0.27. Column 7 shows the weighted, uncorrected number of sources per steradian

$$
N_{\mathrm{w}}=\sum_{i=1}^{N} \frac{1}{\Omega\left(S_{p_{i}}\right)}
$$

with the $1 \sigma$ rms error (Garwood et al. 1988)

$$
\Delta N_{\mathrm{w}}=\sqrt{\sum_{i=1}^{N} \frac{1}{\Omega^{2}\left(S_{p_{i}}\right)}} .
$$

The summation is taken over all sources in the flux density interval. The last column gives the corrected number of sources per flux density interval $\left(S_{\text {range }}\right)$ per steradian normalized to $n_{0}=S_{\mathrm{c}}^{-2.5}$, meaning:

$n \cdot S_{\mathrm{c}}^{2.5}=K \cdot \frac{N_{\mathrm{w}}}{S_{\mathrm{range}}} \cdot S_{\mathrm{c}}^{2.5}$.

The source counts in the LMC snapshot fields are plotted in Fig. 5. The line in the figure represents the extragalactic source count distribution of the Westerbork survey at $1.4 \mathrm{GHz}$ (Oosterbaan 1978). The differential source counts of this survey are well approximated by

$$
n(S)=311 \cdot S^{-2.59-0.106 \cdot \ln S} \mathrm{Sr}^{-1} \mathrm{Jy}^{-1}
$$

The comparison of the ATCA snapshot survey to the Westerbork survey is difficult because the latter is sensitive to more extended structures compared to the ATCA survey. For extended sources we expect that the integrated flux densities of the snapshot sources are underestimated, which causes a higher uncertainty in the source count distribution; for more accurate integrated flux densities we would need more observations with shorter baselines. The LMC source count distribution, however, follows the extragalactic distribution of Oosterbaan very closely. This indicates that most of the compact sources of the LMC survey are background objects. At the highest flux density interval $280 \mathrm{mJy} \leq S_{\mathrm{i}}<560 \mathrm{mJy}$ there are two sources missing compared to the Westerbork survey. This might be due to the underestimated integral flux densities. An excess of sources above the extragalactic source counts is indicated in the flux density range from 8.8 to $35 \mathrm{mJy}$. The discrepency between the number of sources predicted and observed in the 8.8 to $35 \mathrm{mJy}$ bin is only weakly significant, given the uncertainties in flux density in this survey. It suggests, that some of the fainter sources are associated with the LMC, a conclusion strongly supported by the positional coincidence with Halpha knots discussed above.

We get the best match to the extragalactic fit of Oosterbaan (see Fig. 5 lower panel and Table 5) by excluding all 15 possible intrinsic sources in the second and

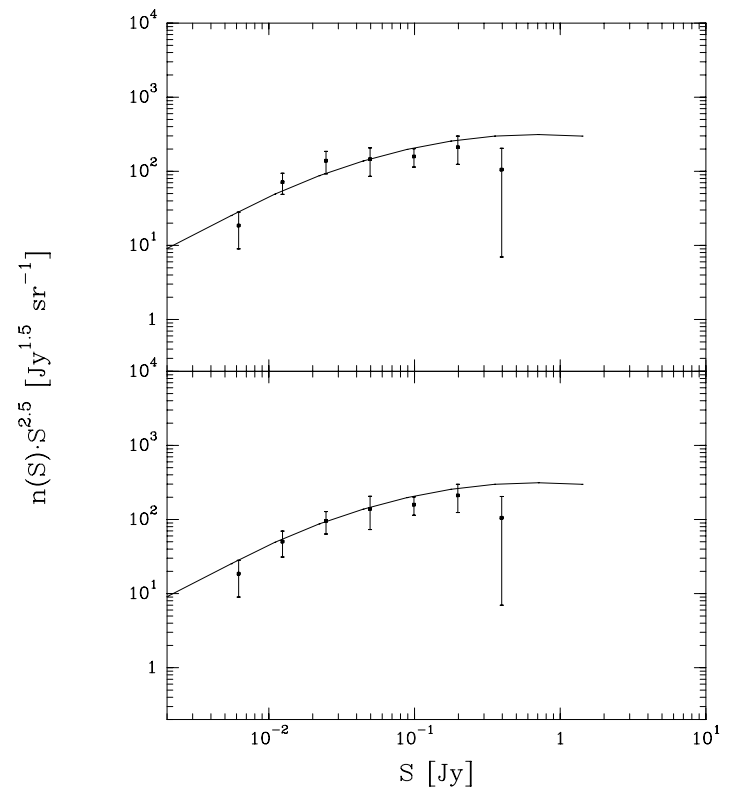

Fig. 5. Source counts for all sources of our survey (top). The line presents the source count distribution of Oosterbaan (1978). The lower panel shows the source counts after excluding all possible intrinsic sources and the known HII regions

third flux density interval for which the position corresponds to a DEM object plus three known HII regions located in the N159 - N160 area (Marx et al., in preparation). So we conclude that only a few compact sources in directions toward the LMC are intrinsic objects, and that most of these have flux densities between 8.8 and $35 \mathrm{mJy}$ and are located within extended $\mathrm{H} \alpha$ emitting regions.

\section{Summary}

A sample of 113 compact radio continuum sources has been detected in and behind the LMC at $1.4 \mathrm{GHz}$ with the Australia Telescope Compact Array (ATCA); 70 objects have been found at $2.4 \mathrm{GHz}$. The sample is almost complete down to a flux density limit of about $6 \mathrm{mJy}$ at $1.4 \mathrm{GHz}$ for sources smaller than $D \sim 54^{\prime \prime}$ and about $3 \mathrm{mJy}$ at $2.4 \mathrm{GHz}$ for $D \sim 34^{\prime \prime}$ (see Table 1 ). As the sizes of the sources are smaller than $13 \mathrm{pc}$ at $1.4 \mathrm{GHz}$ and $8 \mathrm{pc}$ at $2.4 \mathrm{GHz}$ the objects are either extragalactic background sources or compact objects within the LMC, such as compact HII regions or young SNRs. The vast majority of our sources have been seen for the first time. This is due to the higher angular resolution of the ATCA compared to the instruments used for previous surveys.

Excluding the 15 sources which are located in the regions of $\mathrm{H} \alpha$ objects in the Davies et al. (1976) catalogue and which have flux densities in the $8.8-35 \mathrm{mJy}$ range plus three known HII regions, we find that our source counts agree very well with those of Oosterbaan (1978). So we conclude that our catalogue of compact sources contains beyond the 3 known HII regions located in the 
Table 5. Source counts

\begin{tabular}{|c|c|c|c|c|c|c|c|}
\hline \multicolumn{8}{|c|}{ Source counts using all objects } \\
\hline$S_{\mathrm{c}}[\mathrm{Jy}]$ & flux density interval [Jy] & $N_{\text {res }}$ & $N_{\text {unres }}$ & $f_{\text {unres }}$ & K & $10^{-3} N_{\mathrm{w}}\left[\mathrm{sr}^{-1}\right]$ & $n \cdot S^{2.5}\left[\mathrm{sr}^{-1} \mathrm{Jy}^{1.5}\right]$ \\
\hline 0.0062 & $0.0044 \leq S_{\mathrm{i}}<0.0088$ & 0 & 12 & 1 & $3.7 \pm 1.5$ & $7.3 \pm 2.3$ & $18.6 \pm 9.6$ \\
\hline 0.0124 & $0.0088 \leq S_{\mathrm{i}}<0.0175$ & 2 & 28 & 0.93 & $3.5 \pm 0.9$ & $10.5 \pm 1.95$ & $71.5 \pm 22.7$ \\
\hline 0.0247 & $0.0175 \leq S_{\mathrm{i}}<0.0350$ & 3 & 24 & 0.89 & $3.3 \pm 0.9$ & $7.7 \pm 1.5$ & $139.2 \pm 46.8$ \\
\hline 0.0495 & $0.0350 \leq S_{\mathrm{i}}<0.0700$ & 15 & 9 & 0.38 & $1.4 \pm 0.5$ & $6.7 \pm 1.4$ & $146.1 \pm 60.5$ \\
\hline 0.0990 & $0.0700 \leq S_{\mathrm{i}}<0.1400$ & 11 & 2 & 0.15 & & $3.6 \pm 1.0$ & $158.6 \pm 44.1$ \\
\hline 0.1980 & $0.1400 \leq S_{\mathrm{i}}<0.2800$ & 6 & 0 & 0 & & $1.7 \pm 0.7$ & $211.8 \pm 87.2$ \\
\hline 0.3960 & $0.2800 \overline{\leq} S_{\mathrm{i}}<0.5600$ & 1 & 0 & 0 & & $0.3 \pm 0.28$ & $105.7 \pm 98.7$ \\
\hline \multicolumn{8}{|c|}{ Source counts excluding sources with $0.0088 \mathrm{Jy} \leq S_{i}<0.035$ Jy lying within DEM objects } \\
\hline 0.0062 & $0.0044 \leq S_{\mathrm{i}}<0.0088$ & 0 & 12 & 1 & $3.7 \pm 1.5$ & $7.3 \pm 2.3$ & $18.6 \pm 9.6$ \\
\hline 0.0124 & $0.0088 \leq S_{\mathrm{i}}<0.0175$ & 1 & 21 & 0.95 & $3.5 \pm 1.1$ & $7.4 \pm 1.6$ & $50.4 \pm 19.3$ \\
\hline 0.0247 & $0.0175 \leq S_{\mathrm{i}}<0.0350$ & 2 & 16 & 0.89 & $3.3 \pm 0.8$ & $5.3 \pm 1.2$ & $95.8 \pm 31.9$ \\
\hline 0.0495 & $0.0350 \leq S_{\mathrm{i}}<0.0700$ & 14 & 9 & 0.39 & $1.4 \pm 0.6$ & $6.4 \pm 1.33$ & $139.6 \pm 66.5$ \\
\hline 0.0990 & $0.0700 \leq S_{\mathrm{i}}<0.1400$ & 11 & 2 & 0.15 & & $3.6 \pm 1.0$ & $158.6 \pm 44.1$ \\
\hline 0.1980 & $0.1400 \leq S_{\mathrm{i}}<0.2800$ & 6 & 0 & 0 & & $1.7 \pm 0.7$ & $211.8 \pm 87.2$ \\
\hline 0.3960 & $0.2800 \leq \mathrm{S}_{\mathrm{i}}<0.5600$ & 1 & 0 & 0 & & $0.3 \pm 0.28$ & $105.7 \pm 98.7$ \\
\hline
\end{tabular}

N159 - N160 area only about 15 objects which are probably intrinsic to the LMC. More detailed observations of sources within the boundary of DEM objects will be presented in a subsequent paper.

Acknowledgements. We thank the staff of the ATCA for their assistance and advice. We are grateful for the hospitality of the ATCA at Narrabri and Epping during our visit. We thank the members of the Graduiertenkolleg in Bonn/Bochum for very useful discussions. Thanks are especially due to Uwe Herbstmeier for valuable suggestions and information. Jürgen Osterberg and Eva Grebel are thanked for providing us with data for comparisons. The research of M. Marx was supported by the Cusanuswerk (Bonn, Germany), U. Mebold was supported by the Deutsche Forschungsgemeinschaft under grant Me745/16-1. J. Dickey was supported in part by National Science Foundation grants 87-22990 and 92-22130 to the University of Minnesota, by a grant from the NSF Unique Foreign Telescope Fund supervised by the National Radio Astronomy Observatory, and by the Office of International Programs at the University of Minnesota.

\section{References}

Clarke J.N., Little A.G., Mills B.Y., 1976, Aust. J. Phys. Astrophys. Suppl. 40, 1

Clarke B., 1980, A\&A 89, 355

Condon J.J., Condon M.A., 1982, AJ 87, 739

Davies R.D., Elliott K.H., Meaburn J., 1976, Mem. R. Astron. Soc. 81,89
Dickey J.M., Mebold U., Marx M., Amy S., Haynes R.F., Wilson W., 1994, A\&A 289, 357

Downes A.J.B., Longair M.S., Perryman M.A.C., 1981, MNRAS 197, 593

Filipovic M.D., Haynes R.F., White G.L., Jones P.A., Klein U., Wielebinski R., 1995, A\&AS 111, 311

Garwood R.W., Perley R.A., Dickey J.M., Murray M.A., 1988, AJ 96,1655

Habing H.J., Israel F.P., 1979, ARA\&A 17, 345

Hunt M.R., Whiteoak J.B., 1994, Proc. Astron. Soc. Aust. 11, 68

Kennicutt R.C., Bresolin Jr., Bresolin F., Bomans D.J., Bothun G.D., Thompson I.B., 1995, AJ 109, 594

Lortet M.-C., Borde S., Ochsenbein F., 1994, A\&AS 107, 193

McGee R.X., Brooks J.W., Batchelor R.A., 1972a, Aust. J. Phys. 25, 581

McGee R.X., Brooks J.W., Batchelor R.A., 1972b, Aust. J. Phys. 25, 613

Mills B.Y., Turtle A.J., 1984a, Proc. IAU Symp. 108, 283

Mills B.Y., Turtle A.J., Little A.G., Durdin M.J., 1984b, Aust. J. Phys. 37, 321

Oosterbaan C.E., 1978, A\&A 69, 235

Westerlund B.E., Matthewson D.S., 1966, MNRAS 131, 371

Westerlund B.E., 1992, A\&AR 2, 29

Willis A.G., Oosterbaan C.E., De Ruiter H.R., 1976, A\&AS 25,453

Wright A.E., Griffith M., Burke B., Ekers R.D., 1994, ApJS 91, 111 